ARTICLE

https://doi.org/10.1038/s41467-019-13122-4

\title{
Homologous bd oxidases share the same architecture but differ in mechanism
}

\author{
Alexander Theßeling 1,4, Tim Rasmussen ${ }^{2,4}$, Sabrina Burschel ${ }^{1}$, Daniel Wohlwend (1) ${ }^{1}$, Jan Kägi ${ }^{1}$, Rolf Müller ${ }^{3}$, \\ Bettina Böttcher (10) ${ }^{2 \star} \&$ Thorsten Friedrich (1) ${ }^{1 \star}$
}

Cytochrome bd oxidases are terminal reductases of bacterial and archaeal respiratory chains. The enzyme couples the oxidation of ubiquinol or menaquinol with the reduction of dioxygen to water, thus contributing to the generation of the protonmotive force. Here, we determine the structure of the Escherichia coli bd oxidase treated with the specific inhibitor aurachin by cryo-electron microscopy (cryo-EM). The major subunits CydA and CydB are related by a pseudo two fold symmetry. The heme $b$ and $d$ cofactors are found in $\mathrm{CydA}$, while ubiquinone8 is bound at the homologous positions in CydB to stabilize its structure. The architecture of the E. coli enzyme is highly similar to that of Geobacillus thermodenitrificans, however, the positions of heme $b_{595}$ and $d$ are interchanged, and a common oxygen channel is blocked by a fourth subunit and substituted by a more narrow, alternative channel. Thus, with the same overall fold, the homologous enzymes exhibit a different mechanism.

\footnotetext{
${ }^{1}$ Institut für Biochemie, Albert-Ludwigs-Universität, Freiburg, Germany. ${ }^{2}$ Biocenter and Rudolf Virchow Center, Julius-Maximilians-Universität, Würzburg, Germany. ${ }^{3}$ Department of Microbial Natural Products, Helmholtz Institute for Pharmaceutical Research Saarland, Helmholtz Centre for Infection Research and Department of Pharmacy at Saarland University, Saarbrücken, Germany. ${ }^{4}$ These authors contributed equally: Alexander Theßeling, Tim Rasmussen. *email: Bettina.Boettcher@uni-würzburg.de; Friedrich@bio.chemie.uni-freiburg.de
} 
xygenic photosynthesis led to the rise of dioxygen, a strong oxidizing agent, in the atmosphere ${ }^{1,2}$. The use of dioxygen as terminal electron acceptor in respiratory chains enables cells to harvest a maximum amount of redox energy to generate a protonmotive force $(\mathrm{pmf})^{3}$. However, the use of dioxygen may lead to the formation of partly reduced species that are highly cytotoxic and explains the need for sophisticated enzyme mechanisms catalyzing the four-electron reduction of dioxygen to water. Membrane-integrated oxidases use dioxygen as terminal electron acceptor ${ }^{4,5}$. They include the family of cytochrome $b d$ oxidases that are terminal reductases of bacterial and archaeal respiratory chains $s^{4,5}$. The $b d$ oxidases couple quinol oxidation and release of protons to the periplasmic side with proton uptake from the cytoplasmic side to reduce dioxygen to water. Thus, the $b d$ oxidases contribute to the pmf by a vectorial charge transfer 4,5 . They display a high affinity toward dioxygen enabling growth under micro-aerobic conditions. In addition, they are required for a rapid dissociation of gaseous inhibitors, such as nitric oxide and enable growth under oxidative stress conditions in the presence of, e.g., $\mathrm{H}_{2} \mathrm{O}_{2}{ }^{6,7}$. However, the mechanism of $b d$ oxidases is still under debate due to lack of the structure of the Escherichia coli bd-I oxidase, one of the two $b d$ oxidases in this bacterium that has been intensively used for most biochemical and biophysical characterizations ${ }^{8}$.

E. coli $b d$-I oxidase (called $b d$ oxidase for simplicity hereafter) is supposed to consist of two major subunits named CydA and CydB and a small third subunit called CydX. CydA and CydB contain 9 transmembrane (TM) helices each, while CydX is made up of a single-TM helix. CydA harbors all three heme groups of the oxidase, namely the low-spin heme $b_{558}$, serving as electron input device and catalyzing quinol oxidation, the high-spin heme $b_{595}$ that is expected to deliver electrons to the third, the unique d-type heme, which is the site where dioxygen is reduced to water $^{9-11}$. CydA contains a soluble, periplasmic domain, the Qloop that varies in length leading to a "long" Q-loop (e.g., in E. coli) or a "short" Q-loop (e.g., in Geobacillus thermodenitrificans). This domain is expected to be involved in quinone binding and oxidation $^{5,11,12}$. Recently, the structure of the $b d$ oxidase from $G$. thermodenitrificans was obtained at 3.1-4 $\AA$ resolution by $\mathrm{x}$-ray crystallography ${ }^{13}$. Here, we report the structure of the $b d$ oxidase from $E$. coli at $3.3 \AA$ resolution obtained by cryo-electron microscopy (cryo-EM). Our results suggest a different mechanism of the homologous enzymes and might furthermore provide important indications on the development of new antimicrobials that target the $b d$ oxidase of pathogens such as Mycobacteria ${ }^{14,15}$.

\section{Results}

Structure of $\boldsymbol{E}$. coli $\boldsymbol{b} \boldsymbol{d}$ oxidase. The structure of the $E$. coli $b d$ oxidase in the presence of the specific inhibitor aurachin $\mathrm{C}$ was obtained at $3.3 \AA$ resolution by cryo-EM. Details on the cryo-EM procedure, the obtained local resolution and the cryo-EM parameters and statistics are provided in Supplementary Figs. 1 and 2 and in Supplementary Table 1. We were not able to obtain a structure of the E. coli bd oxidase in the absence of the inhibitor although aurachin $\mathrm{C}$ is not detectable in the electron density (see below). The two major subunits of the enzyme, CydA and CydB, share the same fold consisting of 9 TM helices that are arranged as two four-helix bundles and one additional peripheral helix (Fig. 1; Supplementary Fig. 3). The TM domains of both subunits superpose well when applying a twofold rotational symmetry revealing an rmsd of only $4.3 \AA$. This suggests that $c y d A$ and $c y d B$ arose by gene duplication although this is not reflected in the primary sequences (Supplementary Fig. 4). The two subunits interact via hydrophobic residues at the contact sites provided by TM helices 2, 3, and 9 of CydA and the symmetry-related helices
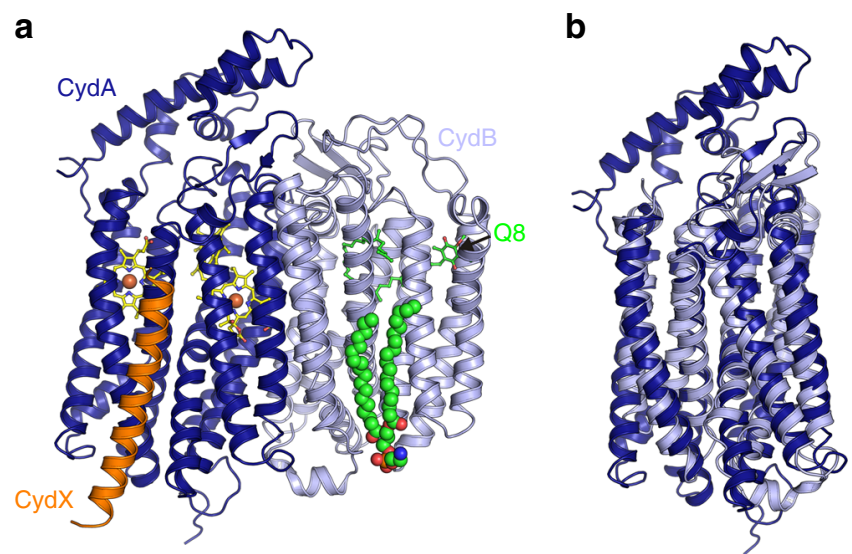

Fig. 1 Cryo-EM structure of $E$. coli bd oxidase at $3.3 \AA$ resolution. a Subunits CydA (dark blue) and CydB (light blue) comprise nine TM helices each. CydX (orange) is a single-TM helix, serving as clamp for the four-helix bundles coordinating hemes $b_{558}$ and $b_{595}$ (shown as sticks). Ubiquinone-8 (Q8) and a glycerophospholipid (shown in spheres) are bound to CydB. b CydA and $C y d B$ share the same fold, highlighting their homology. The extended lid of the $\mathrm{Q}$ binding site at the periplasmic side of the membrane (top), the Q-loop, is unique for CydA

on CydB. The Q-loop of CydA is located on the periplasmic side between TM helices 6 and 7 (Fig. 1; Supplementary Fig. 3) extending over a length of 136 amino acids including the short linkers to the helices (Supplementary Fig. 4). The defined part consists of 5 short helices connected by short loops that form a lid on top of CydA. 40 amino acids of the C-terminus of the Q-loop (positions 262-302) are not resolved in the structure, most likely due to high flexibility, suggesting a hinge function of this region for the lid (Fig. 1). The C-terminus of $\mathrm{CydB}$ forms a small cytoplasmic helix parallel to the membrane plane. In addition, a glycerophospholipid is bound to CydB (Fig. 1). It consists of two C-18 fatty acids as revealed in the structure and ethanolamine as polar head group as determined by TLC analysis (Supplementary Fig. 5) that is held in place by an electrostatic interaction with Arg365. Interestingly, Arg191 is placed just above Arg365 and could probably substitute its function, when binding phospholipids with shorter chain lengths (Supplementary Fig. 5). Both arginine residues are not conserved. The alkyl chains interact with the TM helices $1,2,5,8$, and 9 of CydB by several hydrophobic interactions.

The single helix subunit CydX is made up of 37 residues and interacts with TM helices 1, 5, and 6 of CydA in a stretched conformation (Fig. 1). The C-terminal part of CydX contains mainly hydrophilic amino acids and is exposed to the cytoplasm with Glu25 as a "float" preventing the helix to fully enter the membrane. The $\mathrm{N}$-terminal part of CydX resides within the membrane. Its multiple interactions with CydA might explain that the oxidase is not properly assembled in a $\Delta c y d X \operatorname{strain}^{16}$. Unexpectedly, the E. coli bd oxidase contains a fourth subunit that modeled as a single helical protein with an expected molecular mass of about $3 \mathrm{kDa}$ as deduced from a silver-stained sodium dodecyl sulfate polyacrylamide gel electrophoresis of our preparation (Supplementary Fig. 6). The molecular identity of this subunit was determined by nano-liquid chromatography electrospray ionization mass spectrometry/MS to YnhF, an E. coli protein with one predicted TM helix and located in the cytoplasmic membrane (Supplementary Fig. 6) ${ }^{17-20}$. The protein is produced in conditions of low oxygen concentration ${ }^{17,19}$ and has retained its $\mathrm{N}$-terminal formyl-methionine ${ }^{18}$. The corresponding gene is not part of the cyd-operon encoding the E. coli $b d$ oxidase just consisting of $c y d A, B$, and $X^{16}$, but is located 
a

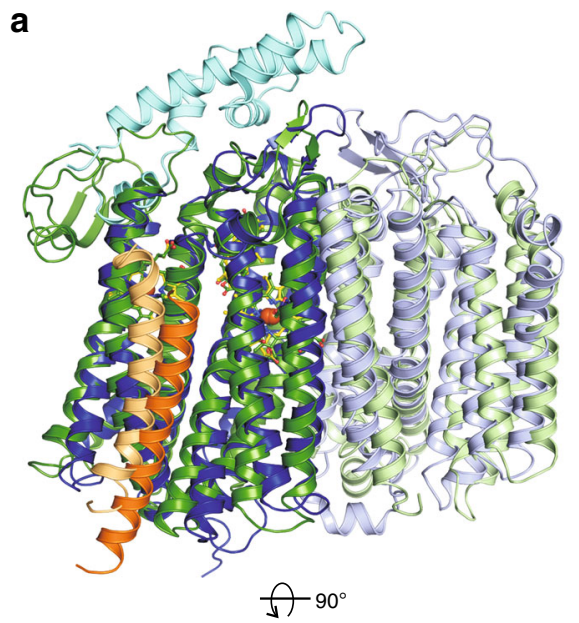

b

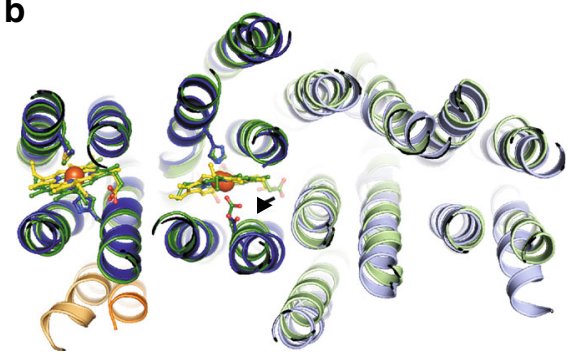

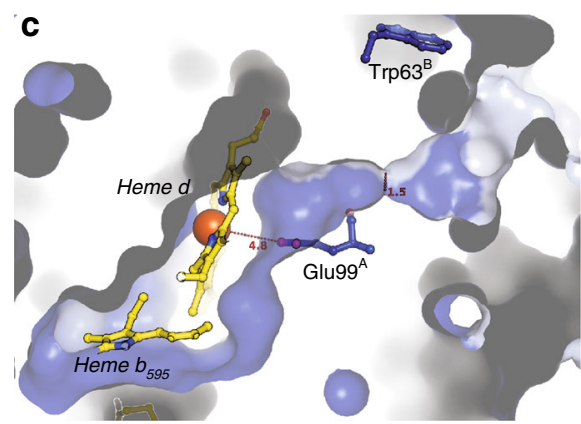

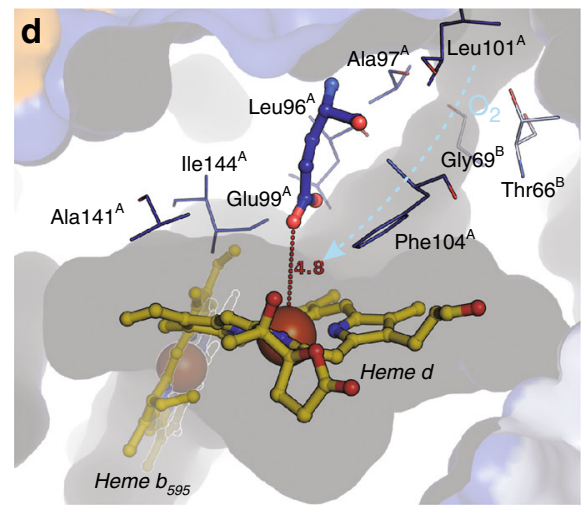

Fig. 2 The heme arrangement in E. coli bd oxidase provides a substrate cavity at heme $d$. a In comparison with the G. thermodenitrificans enzyme (pdb ID 5DOQ, CydA: dark green, CydB: light green, CydS: light orange), the E. coli enzyme features a positional exchange of hemes $b_{595}$ and $d$. $\mathbf{b}$ As opposed to $b_{595}$ of $G$. thermodenitrificans bd oxidase, the central iron of heme $d$ in the E. coli enzyme does not recruit Glu99 of CydA as axial ligand, leaving a voluminous cavity for substrates (arrow). c CydA (dark blue surface) and CydB (light blue surface) form a channel from the solvent to heme $d$ with maximum constriction to $1.5 \AA$. The dislocation of Glu99 (CydA) provides space for a small substrate like dioxygen to bind as axial ligand to heme d. $\mathbf{d}$ Several hydrophobic residues surround Glu99A, forming a hydrophobic roof above the cavity harboring heme $\mathrm{d}$. The putative oxygen channel shown in (c) is indicated with an ice blue arrow. The constriction of this channel is formed by hydrophobic moieties of both CydA (Ala97 and Leu101) and CydB (Thr66 and Gly69)

between $y d h P$ encoding a putative transporter and the transcriptional repressor gene purR ${ }^{17,18}$. We propose to rename the protein to CydY. Noteworthy, a TM helix with stretches homologous to YnhF is also found in several membrane proteins containing more than one TM helix, including eukaryotic membrane proteins. In E. coli, this fourth subunit, CydY, seems to play a crucial role in the mechanism of the $b d$ oxidase (see below).

Comparison to the G. thermodenitrificans enzyme. Unexpectedly $^{21}$, the overall structure of the E. coli oxidase is highly similar to that reported for the G. thermodenitrificans enzyme (Fig. 2) ${ }^{13}$. Remarkably, there are small but substantial differences that presumably result in different mechanisms. Surprisingly, although similarly positioned (Fig. 2$)^{13,22}$, the relative arrangement of the three heme groups in CydA is strikingly different (Fig. 3). They form a triangle with consistent distances between their central iron atoms and they show the same orientation of the heme planes relative to the membrane plane (Figs. 2 and 3). The lowspin $b_{558}$ is unambiguously identified due to the hexacoordination of the central iron by His186 and Met393 and it is positioned below the "lid" provided by the long Q-loop (Fig. 1) as expected from mutagenesis and spectroscopy studies (Fig. 2$)^{8}$. Heme d, however, found closer to the periplasmic side in the $G$. thermodenitrificans structure, and heme $b_{595}$, oriented to the middle of the membrane ${ }^{13}$, occupy interchanged positions in $E$. coli as demonstrated by significantly better density fits (Figs. 2 and 3). The proposed heme arrangement is in line with the spectroscopic characterization of E. coli variants, in which Glu99 and Glu445, the axial ligands to heme $b_{595}$ and $d$, have been mutated $^{8,23-26}$. In our model, heme $b_{595}$ is closer to the periplasmic space and ligated by Glu445 and heme d itself is positioned more to the middle of the membrane with Glu99 as axial ligand. This is backed by the finding that mutating Glu445 indeed had the strongest effects on the ultraviolet (UV)-vis spectra of hemes $b_{595}$ and $b_{558}{ }^{8}$. Further on, it was shown that E. coli Glu445 is critical for the electrochemical properties of heme $b_{595}{ }^{12,23}$. Mutations of Glu99 led to either the loss of the heme $b_{595} /$ heme $d$ active site $24-26,33$, or solely to the loss of heme $d^{8,26}$ implying at least a proximity of Glu99 to heme d. We changed the nonconserved Glu74 ${ }^{\mathrm{A}}$ located at the surface to $\mathrm{CydB}$ to a phenylalanine residue. The residue is located at the level of heme $d$. The sequences of the mutagenic primers are listed in Supplementary Table 2. The E74FA variant was instable and could not be purified but the absorbances for heme $\mathrm{d}$ were lacking in the redox difference of the detergent extract, while a residual absorbance of $\mathrm{b}_{595}$ is clearly detectable supporting our proposed model (Supplementary Fig. 7).

In our model, Glu99 is an axial ligand of heme d. The homolog in G. thermodenitrificans Glu101 has a very short distance of only $2.1 \AA$ to the central $\mathrm{Fe}$ of the positionally equivalent heme $\mathrm{b}_{595}$. In E. coli by contrast, the corresponding Glu99 faces the central Fe of heme $\mathrm{d}$ in an axial distance of $4.8 \AA$, providing space for substrates. The displacement is due to the insertion of Leu101 into TM helix 3 resulting in a stronger curvature (Fig. 2). This provides a voluminous cavity for potential substrates to bind at the axial position of heme $d$, roofed by the hydrophobic Ile144 and Phe104. In fact, dioxygen would fit well into that cavity. This 
a

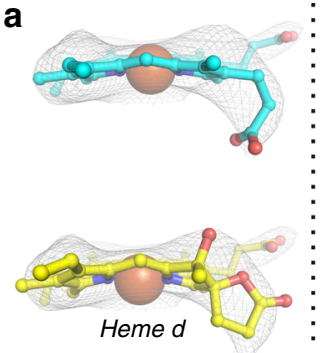

b

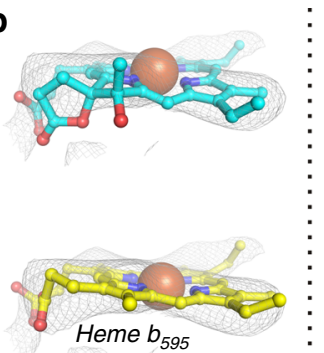

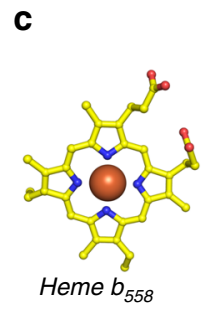

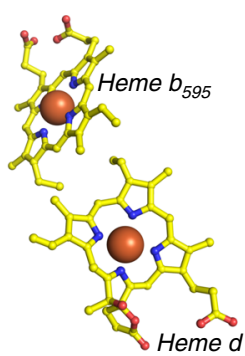

Fig. 3 Heme arrangement in CydA. Electron density maps of $b_{595}$ and d contoured at $1.5 \sigma$ show that, as compared to $G$. thermodenitrificans $b d$ oxidase, $b_{595}$ and d exchange positions in CydA (a, top). Heme $b_{595}$ as found in $G$. thermodenitrificans bd oxidase (blue carbons) does not fit well into the electron density map, neither does heme d (b top). Exchanging positions of both heme groups (yellow carbons) produces substantially better fits of hemes $d$ (a, bottom) and $b_{595}$ (b, bottom), in particular for the hydroxyl group at the spiro substituted pyrrole of heme d. c Arrangement of heme groups in E. coli bd oxidase, periplasm: top of picture, cytoplasm: bottom of picture. The proposed arrangement is in line with the spectroscopic characterization of $E$. coli bd variants, in which Glu99A and Glu445A, both adjacent to the central Fe ion of heme $b_{595}$ and d, respectively, have been mutated ${ }^{8,23-26}$. Mutation of Glu99A, adjacent to heme $d$ according to our model, did not influence the heme $b_{595}$ content, while mutations of Glu445A, adjacent to heme $b_{595}$ according to our model, either influenced solely the content of $b_{595}$ or of both hemes in question $8,23-26$. Mutation of Glu74A, close to heme $d$ according to the proposed model, eliminated the signal of heme d (Supplementary Fig. 7)
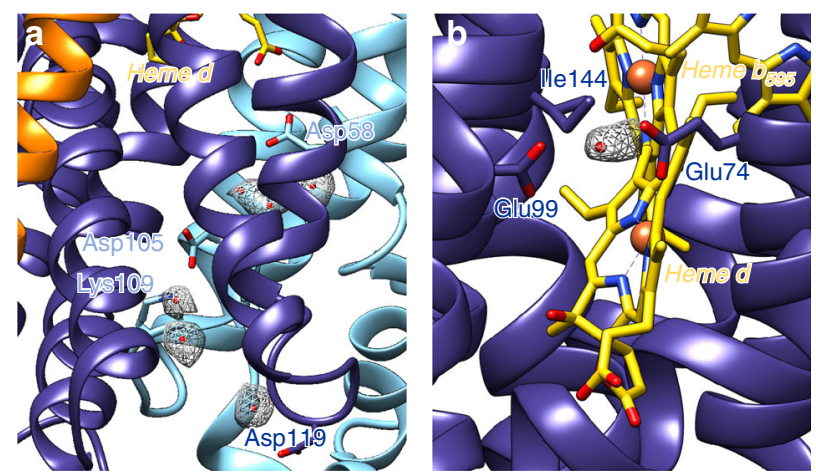

Fig. 4 Water densities within bd oxidase. a A chain of water molecules at the subunit interface was identified providing a proton path from the cytosol to Asp58 on CydB. Protons can be transferred from this residue to the propionate of heme d. $\mathbf{b}$ A density between Glu99 and Glu74 close to heme $\mathrm{d}$ could be caused by the substrate dioxygen or the product water. CydA is shown in dark blue, CydB in light blue and CydX in orange.

Densities of water are shown as meshes

finds support in an extra density that is located in this cavity between E99 and E74, probably caused by a dioxygen or a water molecule (Fig. 4). Further, it was proposed that heme $\mathrm{d}$ is derived from bound heme $\mathrm{b}$ during enzymatic turnover by hydroxylation of the porphyrin ring $^{8}$, requiring protons. However, our model suggests that the proton transfer between Glu99 and Glu445 needed for porphyrin hydroxylation is in fact disrupted (see below). Hence, the b heme coordinated by Glu99 is the sole candidate for hydroxylation in $E$. coli bd oxidase, possibly explaining the different cofactor arrangement.

While heme $b_{558}$ is spatially more separated from hemes $b_{595}$ and $\mathrm{d}$ with $\mathrm{Fe}-\mathrm{Fe}$ distances of 18.6 and $15.2 \AA$, the $\mathrm{Fe}-\mathrm{Fe}$ distance between hemes $b_{595}$ and $d$ is only $11.1 \AA$, in line with previous observations ${ }^{13,27}$. In fact, the shortest edge-to-edge distance between the latter two is only $3.5 \AA$ (Fig. 3) explaining their cooperativity ${ }^{28}$. Trp441 proposed as being liable for donating an electron to the formation of a reaction intermediate, the $\mathrm{F}+{ }^{*}$ state $^{29}$, is found in conserved position in E. coli.

CydB contains a tightly bound ubiquinone- 8 . We found a large electron density extending along hydrophobic clefts in CydB (Fig. 5), perfectly fitting to ubiquinone-8 (Q-8). The quinone
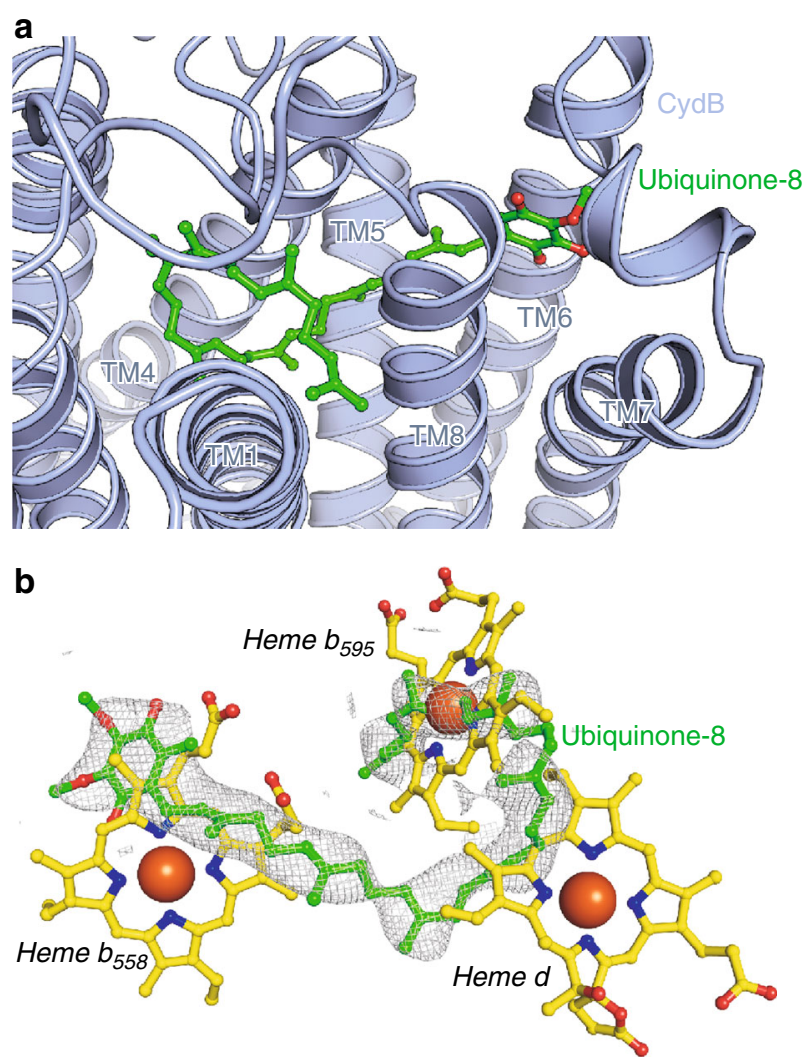

Fig. 5 E. coli bd oxidase features a Q-8 binding site in CydB that has a stabilizing function. a Ubiquinone-8 (shown as sticks) binds to subunit CydB (light blue). It deeply inserts into CydB, involving six TM helices in binding. $\mathbf{b}$ A superposition of $C y d A$ and $C y d B$ shows that in $C y d B$, ubiquinone- 8 follows the trace of the heme positions in CydA

head group points away from CydA into the membrane space, indicating a structural rather than a functional role of Q-8 (Fig. 5). The overlay of CydA and CydB shows that Q-8 follows the arrangement of the heme groups in CydA. We examined the role of bound Q-8 by mutating conserved alanine residues at the surface of the hydrophobic cleft to phenylalanine residues that might disturb binding of Q- 8 . The membranes from the $\mathrm{A} 82 \mathrm{~F}^{\mathrm{B}}$, $\mathrm{A} 137 \mathrm{~F}^{\mathrm{B}}$, and the $\mathrm{A} 172 \mathrm{~F}^{\mathrm{B}}$ mutants showed 80 to $70 \%$ of the 
NADH oxidase activity of the membranes of the parental strain. We attributed the lower activity to a hindered Q-8 binding supporting the idea that bound Q-8 stabilizes CydB by substituting the heme groups. Remarkably, the excess aurachin $\mathrm{C}$ did not substitute the Q-8 molecule indicating its tight binding.

Binding of aurachin. Aurachins prevent the binding of the quinol substrate to the oxidases ${ }^{30-32}$. We determined the apparent $\mathrm{IC}_{50}$ of aurachins $\mathrm{C}$ and $\mathrm{D}$ towards the duroquinol:dioxygen oxidoreductase activity of our preparation to 12 and $35 \mathrm{nM}$, respectively, emphasizing its specific binding (Supplementary Fig. 7). Due to its high affinity towards the $b d$ oxidase, we incubated the preparation with $1 \mu \mathrm{M}$ aurachin $\mathrm{C}$ prior to cryoEM. Although we obtained the structure of the E. coli bd oxidase only in the presence of aurachin C, we unexpectedly found only little density assignable to aurachin at the proposed quinol binding site, including Glu257 and Lys252 of CydA $\mathrm{A}^{5,11,12,33}$. Most likely, the flexibility of the Q-loop above the binding site prohibits a reliable assignment.

The oxygen entry site. Heme $b_{595}$ is directly connected to the protein surface by a solvent accessible substrate channel also identified in the G. thermodenitrificans enzyme (Supplementary Fig. 8$)^{13}$. Noteworthy, in $E$. coli the cavity extends to $b_{595}$ but not to heme $d$ that binds dioxygen. A deeper penetration of dioxygen into that channel towards heme $\mathrm{d}$ is blocked by heme $\mathrm{d}$ itself. Thus, as dioxygen has to bind to heme $\mathrm{d}$ and not to $\mathrm{b}_{595}$ the conserved access to the latter has to be inaccessible in E. coli oxidase. Indeed, a density is detectable at this position that modeled as a single helical structure and that was identified as CydY (Supplementary Fig. 6). It is proposed that CydY is a fourth subunit of the E. coli bd oxidase blocking the dioxygen channel present in both enzymes. An alternative pathway to guide dioxygen to heme $\mathrm{d}$ not present in the G. thermodenitrificans enzyme is provided by a small hydrophobic channel starting above Trp63 on CydB and extending further to heme $\mathrm{d}$ on CydA (Fig. 2). The E. coli specific channel has a constriction with a diameter of about $1.5 \AA$ that might act as a selectivity filter. While dioxygen might easily pass through the constriction, angled molecules such as hydrogen sulfide may not.

Proton pathways. The reaction of the $b d$ oxidase is coupled with the generation of a pmf due to proton uptake from the cytoplasmic side to reduce dioxygen to water while protons released by quinol oxidation are delivered to the periplasmic side. Several proton pathways from the cytoplasmic side to the active site have been proposed based on the structure of the G. thermodenitrificans enzyme and by mutagenesis studies using the $E$. coli enzyme $8,13,23,24,33,34$. Our structure shows a water chain along a hydrophilic channel for proton transfer, entering the enzyme at D119 ${ }^{\mathrm{A}}$, along $\mathrm{K} 57^{\mathrm{A}}, \mathrm{K}_{109^{\mathrm{B}}}, \mathrm{D} 105^{\mathrm{B}}, \mathrm{Y} 379^{\mathrm{B}}$ to $\mathrm{D} 58^{\mathrm{B}}$ (Fig. 4). From here protons can be transferred to the propionate group of heme d. In G. thermodenitrificans, the proton has to be transferred further to the homolog of E. coli Glu445, the ligand of heme $\mathrm{d}$, potentially involving the heme $\mathrm{d}$ propionate. This proton transfer is no longer required in the E. coli $b d$ oxidase and accordingly blocked by Ile144 that acts as a hydrophobic separator.

\section{Discussion}

The $b d$ oxidases from $E$. coli and G. thermodenitrificans are homologous enzymes (Supplementary Fig. 4) and adopt a highly similar architecture (Fig. 2). However, the position of the hemes $b_{595}$ and $d$ are interchanged leading to a different position of the binding site for the gaseous substrates (Figs. 2 and
3). One spacious entry site for the gaseous substrates is conserved between the two enzymes, however, blocked by a fourth subunit, CydY, in the E. coli enzyme (Supplementary Fig. 6). The second binding site only present in the E. coli enzyme is accessible by a long channel that contains a constriction possibly conferring substrate specificity. Together with the low activity of the G. thermodenitrificans ${ }^{13}$ enzyme as compared to the E. coli enzyme with high turnover ${ }^{35}$ this indicates that the former enzyme is predominantly involved in the detoxification of the bacterium's environment, while the latter seems to act as a true oxidase. The presence of $c y d Y$ (or formerly $y n h F$ ) encoding the fourth subunit might therefore be used as a marker to identify the molecular tasks of the homologous enzymes with different mechanisms.

\section{Methods}

Preparation of bd oxidase from E. coli BL21 ${ }^{\star} \Delta$ cyo/pET28a $c y d A_{h} B X$. An E. coli strain lacking the chromosomal copy of the cyo genes encoding $b o_{3}$ oxidase was used. The strain was transformed with pET28a $c y d A_{h} B X^{16}$. All steps to purify $b d$ oxidase were carried out at $4{ }^{\circ} \mathrm{C}$. Frozen cells (35-40 g wet weight) were suspended in the 6-fold volume $20 \mathrm{mM} 3$-(N-morpholino)propanesulfonic acid (MOPS), $20 \mathrm{mM} \mathrm{NaCl}, 1 \mathrm{mM}$ phenylmethylsulfonyl fluoride (PMSF), pH 7.0 and disrupted by single pass through a French pressure cell (SLM Aminco) at 16,000 psi. Cell debris were removed by centrifugation at $12,000 \times g$ for $20 \mathrm{~min}$. Cytoplasmic membranes were sedimented by ultracentrifugation of the cleared lysate at $250,000 \times g$ for $75 \mathrm{~min}$ and suspended in $20 \mathrm{mM}$ MOPS, $20 \mathrm{mM} \mathrm{NaCl}, 0.5 \mathrm{mM}$ PMSF, pH 7.0 to a final protein concentration of $10 \mathrm{mg} / \mathrm{mL}$. Membrane proteins were solubilized by incubating the membrane suspension $1 \mathrm{~h}$ with $1 \%$ Lauryl Maltose Neopentyl Glycol (BioFroxx) stirring at $4^{\circ} \mathrm{C}$. Nonsolubilized material was separated by ultracentrifugation at $250,000 \times g$ for $15 \mathrm{~min}$. The supernatant was applied to a $25 \mathrm{ml}$ HisTrapFF (GE Healthcare) column pre-equilibrated in $50 \mathrm{mM}$ MOPS, $500 \mathrm{mM} \mathrm{NaCl}, 50 \mathrm{mM}$ imidazole, $0.5 \mathrm{mM}$ PMSF, 0.003\% MNG, pH 7.0, with a flow rate of $2 \mathrm{~mL} / \mathrm{min}$. The column was washed with buffer containing $68 \mathrm{mM}$ imidazole until the UV-baseline dropped to the original value. Bound proteins were eluted in a linear gradient of $3 \mathrm{CV}$ from 68 to $500 \mathrm{mM}$ imidazole. Peak fractions were pooled and washed two times with buffer ( $20 \mathrm{mM}$ MOPS, $20 \mathrm{mM} \mathrm{NaCl}, 0.5 \mathrm{mM}$ PMSF, pH 7.0). The concentrated sample was then applied onto a MonoQ 10/100 GL ion exchange column (GE Healthcare) in $20 \mathrm{mM}$ MOPS, $20 \mathrm{mM} \mathrm{NaCl}, 0.5 \mathrm{mM}$ PMSF, pH 7.0 and eluted in a gradient of $10 \mathrm{CV}$ up to $350 \mathrm{mM} \mathrm{NaCl}$. The concentrated sample was then applied onto a Superose 6 Inc 10/300 GL (GE Healthcare) size-exclusion chromatography column equilibrated in buffer (20 mM MOPS, $20 \mathrm{mM} \mathrm{NaCl}, 0.5 \mathrm{mM}$ PMSF, pH 7.0) at a flow rate of $0.2 \mathrm{~mL} / \mathrm{min}$. Peak fractions were pooled and used for further analysis. For cryo-EM the samples were treated with $1 \mu \mathrm{M}$ aurachin $\mathrm{C}$.

Cryo-electron microscopy and data analysis. $3.5 \mu \mathrm{l}$ of $2 \mathrm{mg} / \mathrm{mL} b d$ oxidase in amphipol A8-35 was applied to a glow-discharged, holey carbon coated copper 400 grid R1.2/1.3 (Quantifoil) and vitrified in liquid ethane with a Vitrobot mark IV (FEI) with following settings: $3.5 \mathrm{~s}$ blot time, 5 blot force, $0 \mathrm{~s}$ drain and waiting time, $100 \%$ humidity at $4{ }^{\circ} \mathrm{C}$. Data were collected at the Würzburg cryoEM facility on a Titan Krios G3 (Thermo Scientific) equipped with a Falcon III direct detector. The magnification was set to 75,000 (calibrated pixel size 1.0635 $\AA /$ pixel), the condenser aperture to $70 \mu \mathrm{m}$, and the objective aperture to $100 \mu \mathrm{m}$. Movies with 47 fractions were recorded in counting mode at parallel illumination with a total exposure of $59 \mathrm{e}^{-} / \AA^{2}$. A defocus range of $1.4-2.2 \mu \mathrm{m}$ was applied. Movies were motion corrected and dose weighted with the program MotionCor $2^{36}$ and the contrast transfer functions were obtained with the program CTFFind $4^{37}$. The data were further processed with the program Relion 3.0 $0^{38,39}$. Manual particle picking and 2D classification provided templates for auto-picking in Relion. The particle set was cleaned up with two rounds of $2 \mathrm{D}$ classification. An initial model was obtained with the InitialModel option of relion_refine and thereafter used for the auto-refinement. Two rounds of 3D classification provided the final particle set which was used for a final autorefinement. The local resolution was estimated with the locres option or relion_postprocess. The program Coot $0.8 .9 .1^{40}$ was used for model building using the crystal structure of $G$. thermodenitrificans bd oxidase (pdb: 5DOQ) as starting model. The model was refined with the program Phenix realspace.refine version 1.13.2998 11,42 and validated with MolProbity $4.4^{43}$. Images were prepared with the programs Chimera $1.11^{44}$ and PyMOL 1.8.

Determination of the polar head group of bound phospholipids. $500 \mu \mathrm{L}$ of a $E$. coli bd oxidase preparation $(20 \mathrm{mg} / \mathrm{mL})$ was mixed with $1 \mathrm{~mL}$ chloroform/ methanol $(2: 1, \mathrm{v} / \mathrm{v})$ and incubated at RT for $45 \mathrm{~min} .200 \mu \mathrm{L}$ pure water was added and the suspension was mixed again. After centrifugation (1 min, RT, $1000 \mathrm{rcf}$ ) the organic phase was separated and evaporated in a nitrogen flow. The residual was dissolved in $100 \mu \mathrm{L}$ chloroform. $5 \mu \mathrm{L}$ of the concentrated organic phase and of 
standard solutions of phosphatidylglycerol $(0.013 \mathrm{M})$, phosphatidylethanolamine $(0.015 \mathrm{M})$, phosphatidylserine $(0.012 \mathrm{M})$ and phosphatidylcholine $(0.032 \mathrm{M}$, all from Avanti, Alabaster, Alabama) were placed on the TLC plate (silica gel 60 F254, $20 \times 20 \mathrm{~cm}$, Merck, Darmstadt, and dried for $5 \mathrm{~min}$ at $60^{\circ} \mathrm{C}$. The plate was placed into a TLC chamber, filled with chloroform-methanol-water $(75: 25: 2.5, \mathrm{v} / \mathrm{v} / \mathrm{v})$ as mobile phase. The separation was stopped when the front of the mobile phase was $0.5 \mathrm{~cm}$ below the end of the plate. The plate was dried in a drying cabinet for $5 \mathrm{~min}$ at $60^{\circ} \mathrm{C}$. TLC plates were developed by spraying with primuline solution and drying for $5 \mathrm{~min}$ at $60^{\circ} \mathrm{C}$. Phosphorylated TLC-spots were detected with a Fluorescence imager (Fusion SL, Vilber Lourmat) using a Blue535Y filter and $80 \mathrm{~ms}$ detection time.

Amphipol exchange. To replace the detergent MNG, isolated $b d$ oxidase was supplemented with a threefold mass excess of amphipol A8-35 (Anatrace, Maumee, Ohio). The sample was incubated at $4^{\circ} \mathrm{C}$ for 90 min while stirring. The excess detergent was removed by addition of 40 fold mass excess BioBeads (SM-2 resin, BioRad) and further $3 \mathrm{~h}$ incubation at $4{ }^{\circ} \mathrm{C}$ under mild stirring. To remove excess amphipols, the sample was loaded onto a Superose 6 Increase $(24 \mathrm{~mL})$ size-exclusion column equilibrated in $20 \mathrm{mM}$ MOPS, pH 7.0, $20 \mathrm{mM} \mathrm{NaCl}$. The homogenous peak fractions contained the enzyme in amphipol A8-35 without detergent.

Inhibition of bd oxidase by aurachin C and $\mathbf{D}$. The inhibitory potency of aurachin $\mathrm{C}$ and $\mathrm{D}$ on the isolated $E$. coli $b d$ oxidase was determined by measuring the duroquinol:oxygen oxidoreductase activity ${ }^{35} .10 \mu \mathrm{L}$ of a $1 \mathrm{M}$ DTT solution prepared under anoxic conditions were injected in the chamber of an oxygen electrode (oxygraph+, Hansatech) in $2 \mathrm{~mL}$ buffer (20 mM MOPS pH 7.0, $20 \mathrm{mM} \mathrm{NaCl}$, $0.003 \% \mathrm{MNG}$ ) at $30^{\circ} \mathrm{C} .5 \mu \mathrm{L}$ of a $100 \mathrm{mM}$ of duroquinone (Sigma) in ethanol were added. The reaction was started by an addition of $1 \mu \mathrm{l} b d$ oxidase $(1 \mathrm{mg} / \mathrm{mL})$. Various amounts of aurachins $\mathrm{C}$ and $\mathrm{D}$ were added to the reaction. The nonenzymatic reaction was less than $1 \%$ of the rate of the enzymatic reaction. The enzymatic rate was corrected for the non-enzymatic one. Each data point was assayed in triplicates.

Reporting summary. Further information on research design is available in the Nature Research Reporting Summary linked to this article.

\section{Data availability}

The cryo-EM maps have been deposited in the Electron Microscopy Data Bank (EMDB) under the accession number EMD-10049. The atomic model has been deposited in the Protein Data Bank under accession number 6RX4. The source data underlying Supplementary Figs. 5b, 6b 7a, 7b, and 9d are provided as a Source Data file. Other data are available from the corresponding authors upon reasonable request.

Received: 9 July 2019; Accepted: 22 October 2019;

Published online: 13 November 2019

\section{References}

1. Bailey, D. M. Making sense of oxygen; quantum leaps with 'physics-iology'. Exp. Physiol. 104, 453-457 (2019).

2. Ducluzeau, A. L. et al. Was nitric oxide the first deep electron sink? Trends Biochem. Sci. 34, 9-15 (2009).

3. Mitchell, P. Coupling of phosphorylation to electron and hydrogen transfer by a chemi- osmotic type of mechanism. Nature 191, 144-148 (1961).

4. Hemp, J. \& Gennis, R. B. Diversity of the heme-copper superfamily in archaea: insights from genomics and structural modeling. Results Probl. Cell Differ. 45, 1-31 (2008).

5. Borisov, V. B., Gennis, R. B., Hemp, J. \& Verkhovsky, M. I. The cytochrome bd respiratory oxygen reductases. Biochim. Biophys. Acta 1807, 1398-1413 (2011).

6. Giuffrè, A., Borisov, V. B., Arese, M., Sarti, P. \& Forte, E. Cytochrome bd oxidase and bacterial tolerance to oxidative and nitrosative stress. Biochim. Biophys. Acta 1837, 1178-1187 (2014).

7. Forte, E., Borisov, V. B., Vicente, J. B. \& Giuffrè, A. Cytochrome bd and gaseous ligands in bacterial physiology. Adv. Microb. Physiol. 71, 171-234 (2017).

8. Murali, R. \& Gennis, R. B. Functional importance of glutamate-445 and glutamate- 99 in proton-coupled electron transfer during oxygen reduction by cytochrome bd from Escherichia coli. Biochim. Biophys. Acta 1859, 577-590 (2018).

9. Miyoshi, H., Takegami, K., Sakamoto, K., Mogi, T. \& Iwamura, H. Characterization of the ubiquinol oxidation sites in cytochromes bo and $b d$ from Escherichia coli using aurachin C analogues. J. Biochem. 125, 138-142 (1999).
10. Mogi, T. et al. Probing the ubiquinol-binding site in cytochrome $b d$ by sitedirected mutagenesis. Biochemistry 45, 7924-7930 (2006).

11. Zhang, J. et al. Site-directed mutation of the highly conserved region near the Q-loop of the cytochrome $b d$ quinol oxidase from Escherichia coli specifically perturbs heme b595. Biochemistry 40, 8548-8556 (2001).

12. Zhang, J., Hellwig, P., Osborne, J. P. \& Gennis, R. B. Arginine 391 in subunit I of the cytochrome $b d$ quinol oxidase from Escherichia coli stabilizes the reduced form of the hemes and is essential for quinol oxidase activity. J. Biol. Chem. 279, 53980-53987 (2004).

13. Safarian, S. et al. Structure of a $b d$ oxidase indicates similar mechanisms for membrane-integrated oxygen reductases. Science 352, 583-586 (2016).

14. Hards, K. \& Cook, G. M. Targeting bacterial energetics to produce new antimicrobials. Drug Resist. Updates 36, 1-12 (2018).

15. Bald, D., Villellas, C., Lu, P. \& Koul, A. Targeting energy metabolism in Mycobacterium tuberculosis, a new paradigm in antimycobacterial drug discovery. MBio 8, e00272-17 (2017).

16. Hoeser, J., Hong, S., Gehmann, G., Gennis, R. B. \& Friedrich, T. Subunit CydX of Escherichia coli cytochrome $b d$ ubiquinol oxidase is essential for assembly and stability of the di-heme active site. FEBS Lett. 588, 1537-1541 (2014).

17. Hemm, M. R., Paul, B. J., Schneider, T. D., Storz, G. \& Rudd, K. E. Small membrane proteins found by comparative genomics and ribosome binding site models. Mol. Microbiol. 70, 1487-1501 (2008).

18. Guan, Z., Wang, X. \& Raetz, C. R. H. Identification of a chloroform-soluble membrane miniprotein in Escherichia coli and its homolog in Salmonella typhimurium. Anal. Biochem. 409, 284-289 (2011).

19. Hemm, M. R. et al. Small stress response proteins in Escherichia coli: proteins missed by classical proteomic studies. J. Bacteriol. 192, 46-58 (2010).

20. Fontaine, F., Fuchs, R. T. \& Storz, G. Membrane localization of small proteins in Escherichia coli. J. Biol. Chem. 286, 32464-32474 (2011).

21. Cook, G. M. \& Poole, R. K. A bacterial oxidase like no other? Science 352, 518-519 (2016).

22. Ingledew, W. J., Rothery, R. A., Gennis, R. B. \& Salerno, J. C. The orientation of the three haems of the 'in situ' ubiquinol oxidase, cytochrome $b d$, of Escherichia coli. Biochem. J. 282, 255-259 (1992).

23. Belevich, I. et al. Time-resolved electrometric and optical studies on cytochrome $b d$ suggest a mechanism of electron-proton coupling in the diheme active site. Proc. Natl Acad. Sci. USA 102, 3657-3662 (2005).

24. Fang, H. F., Lin, R.-J. \& Gennis, R. B. Location of heme axial ligands in the cytochrome d terminal oxidase complex of Escherichia coli determined by sitedirected mutagenesis. J. Biol. Chem. 264, 8026-8032 (1989).

25. Mogi, T., Endou, S., Akimoto, S., Morimoto-Tadokoro, M. \& Miyoshi, H. Glutamates 99 and 107 in transmembrane helix III of subunit I of cytochrome bd are critical for binding of the heme b595-d binuclear center and enzyme activity. Biochemistry 45, 15785-15792 (2006).

26. Mogi, T. Probing the haem d-binding site in cytochrome bd quinol oxidase by site-directed mutagenesis. J. Biochem. 145, 763-770 (2009).

27. Arutyunyan, A. M. et al. Strong excitonic interactions in the oxygen-reducing site of bd-type oxidase: the Fe-to-Fe distance between hemes $\mathrm{d}_{\text {and }} \mathrm{b}_{595}$ is 10 A. Biochemistry 47, 1752-1759 (2008).

28. Jasaitis, A. et al. Electrogenic reactions of cytochrome bd. Biochemistry 39, 13800-13809 (2000)

29. Paulus, A., Rossius, S. G., Dijk, M. \& de Vries, S. Oxoferryl-porphyrin radical catalytic intermediate in cytochrome $b d$ oxidases protects cells from formation of reactive oxygen species. J. Biol. Chem. 287, 8830-8838 (2012).

30. Kunze, B., Höfle, G. \& Reichenbach, H. The aurachins, new quinoline antibiotics from myxobacteria: production, physico-chemical and biological properties. J. Antibiot. 40, 258-265 (1987).

31. Jünemann, S., Wrigglesworth, J. M. \& Rich, P. R. Effects of decyl-aurachin D and reversed electron transfer in cytochrome bd. Biochemistry 36, 9323-9331 (1997).

32. Meunier, B., Madgwick, S. A., Reil, E., Oettmeier, W. \& Rich, P. R. New inhibitors of the quinol oxidation sites of bacterial cytochromes $b o$ and $b d$. Biochemistry 34, 1076-1083 (1995).

33. Yang, K. et al. Glutamate 107 in subunit I of the cytochrome $b d$ quinol oxidase from Escherichia coli is protonated and near the heme $\mathrm{d} / \mathrm{heme} \mathrm{b}_{595}$ binuclear center. Biochemistry 46, 3270-3278 (2007).

34. Borisov, V. B., Belevich, I., Bloch, D. A., Mogi, T. \& Verkhovsky, M. I. Glutamate 107 in subunit I of cytochrome $b d$ from Escherichia coli is part of a transmembrane intraprotein pathway conducting protons from the cytoplasm to the heme $b_{595} /$ heme d active site. Biochemistry 47, 7907-7914 (2008).

35. Al-Attar, S. et al. Cytochrome $b d$ displays significant quinol peroxidase activity. Sci. Rep. 6, 27631 (2016).

36. Zheng, S. Q. et al. MotionCor2: anisotropic correction of beam-induced motion for improved cryo-electron microscopy. Nat. Methods 14, 331-332 (2017).

37. Rohou, A. \& Grigorieff, N. CTFFIND4: fast and accurate defocus estimation from electron micrographs. J. Struct. Biol. 192, 216-221 (2015). 
38. Scheres, S. H. W. RELION: implementation of a Bayesian approach to cryoEM structure determination. J. Struct. Biol. 180, 519-530 (2012).

39. Zivanov, J. et al. New tools for automated high-resolution cryo-EM structure determination in RELION-3. Elife 7, 42166 (2018).

40. Emsley, P., Lohkamp, B., Scott, W. G. \& Cowtan, K. Features and development of Coot. Acta Crystallogr. Sect. D Biol. Crystallogr. 66, 486-501 (2010).

41. Adams, P. D. et al. PHENIX: a comprehensive Python-based system for macromolecular structure solution. Acta Crystallogr. Sect. D Biol. Crystallogr. 66, 213-221 (2010).

42. Afonine, P. V., Grosse-Kunstleve, R. W., Adams, P. D. \& Urzhumtsev, A Bulk-solvent and overall scaling revisited: faster calculations, improved results. Acta Crystallogr. Sect. D Biol. Crystallogr. 69, 625-634 (2013).

43. Chen, V. B. et al. MolProbity: all-atom structure validation for macromolecular crystallography. Acta Crystallogr. Sect. D Biol. Crystallogr. 66, 12-21 (2010).

44. Pettersen, E. F. et al. UCSF Chimera-a visualization system for exploratory research and analysis. J. Comput. Chem. 25, 1605-1612 (2004).

\section{Acknowledgements}

We thank Drs. Schara Safarian and Alexander Hahn, Frankfurt, for most helpful discussions and sharing unpublished data. Electron microscopic data were acquired at the cryo-EM facility of the University Würzburg (DFG equipment grant INST 93/903-1 FUGG). This work was supported by the Deutsche Forschungsgemeinschaft by grants278002225/RTG 2202 and 235777276/RTG 1976.

\section{Author contributions}

A.T. and J.K. prepared the enzyme and performed the amphipol exchange, A.T. performed enzyme kinetic measurements and determined the identity of the lipid, T.R. and A.T. made the EM grids, T.R. and B.B. measured the samples, solved the structure, and refined the model, S.B. made and grew mutants, A.T. and S.B. recorded UV-vis spectra, R.M. provided the aurachins, D.W., T.R., A.T., and B.B. made the figures, T.F. designed the project and wrote the paper with corrections from all co-authors.

\section{Competing interests}

The authors declare no competing interests.

\section{Additional information}

Supplementary information is available for this paper at https://doi.org/10.1038/s41467 019-13122-4.

Correspondence and requests for materials should be addressed to B.Böt. or T.F.

Peer review information Nature Communications thanks Dirk Bald and the other, anonymous, reviewer(s) for their contribution to the peer review of this work. Peer reviewer reports are available.

Reprints and permission information is available at http://www.nature.com/reprints

Publisher's note Springer Nature remains neutral with regard to jurisdictional claims in published maps and institutional affiliations.

(c) Open Access This article is licensed under a Creative Commons Attribution 4.0 International License, which permits use, sharing, adaptation, distribution and reproduction in any medium or format, as long as you give appropriate credit to the original author(s) and the source, provide a link to the Creative Commons license, and indicate if changes were made. The images or other third party material in this article are included in the article's Creative Commons license, unless indicated otherwise in a credit line to the material. If material is not included in the article's Creative Commons license and your intended use is not permitted by statutory regulation or exceeds the permitted use, you will need to obtain permission directly from the copyright holder. To view a copy of this license, visit http://creativecommons.org/ licenses/by/4.0/.

(c) The Author(s) 2019 